\title{
Proposal of a Method to Detect Size of Overlapping Cardboard Boxes Using Plural Lightings
}

\author{
Ryota Iwaizono, Shiyuan Yang, Siichi Serikawa \\ Department of Electrical and Electronic Engineering, Kyushu Institute of Technology, Kitakyushu, Japan \\ *Corresponding Author: iwaizono.ryouta849@mail.kyutech.jp
}

\begin{abstract}
Currently, due to the rapid increase in demand for home delivery, the introduction of automatic guided vehicles in a warehouse is spreading all over the world. However, when an automated guided vehicle drops luggage, it becomes a big problem. In order to prevent this, the automatic guided vehicle is required to automatically control the speed according to the state of the loaded luggage. For that, we need to know the placement and size of the luggage. However, it is difficult to measure it automatically. Cardboard boxes are often used as luggage for home delivery. When cardboard boxes overlap on vehicle, it is difficult to distinguish them and detect them. Because the color of cardboard boxes are similar. Therefore, it is necessary to prepare multiple high-performance cameras for the detection, which is costly. In addition, the detection accuracy is also not high.

In order to solve this problem, we propose a method to detect the size and position of a cardboard box using four lights. In this study, four lights are attached on a vehicle. By overlapping images from 4 directions, there is a shadow even when cardboard boxes are overlapped. This easily extracts the edge of the cardboard box. From the extracted edge, we can detect the size of the cardboard box. For the validity of our method, we practiced experiments. The cardboard boxes are all the same color. As a result, even if the cardboard box is overlapped, we can detect the size and positon of a box correctly.
\end{abstract}

Keywords: Detection, Measurement, Size, Overlapping, Image processing, Automatic guided vehicle, Illumination

\section{Introduction}

In recent years, the reduction of the working population has become a big issue. Recently, the demand for courier services is expanding rapidly all over the world, and due to the decrease in the working population, the spread of automated guided vehicles is rapidly introduced. However, there is a problem that it drops luggage. As a representative method for preventing them, there are cases where a stopper is attached and it is fixed by a rope. However, these methods require human hands. Therefore, the introduction is not widespread. In order to solve these problems, it is expected for an automatic guided vehicles to automatically measure the position and size of luggage and make optimum driving according to the state of luggage. There are many methods to measure the size of an object(1)(2). As representative methods, there are measurements by three-dimensional scanner(3)(4) and measurement by image processing(5)(6). Both methods can detect the size of the object with high accuracy, but they cost. In addition, someone is needed for measurement. As a result, these methods cannot be used with automatic guided vehicles. In addition, the method by image processing has a technical problem. Cardboard box is often used as package for delivery service. They are stacked on a vehicle. Because the color of cardboard is almost the same color, it is very difficult to distinguish them by image processing.

In order to solve these problems, a new method is proposed in this study. Using the four illuminations and a camera, even if cardboard boxes of the same color are stacked, we can detect the size and position of the cardboard box.

\section{Method to detect size and positon}




\section{1}

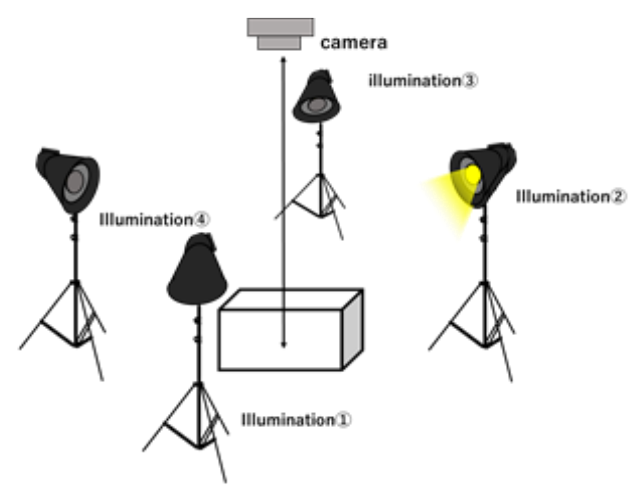

Figure 1:An outline of system configuration in this study.

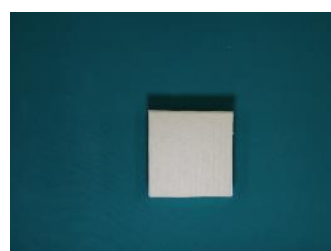

(a) Illumination 1

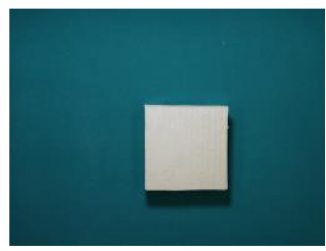

(c) Illumination 3

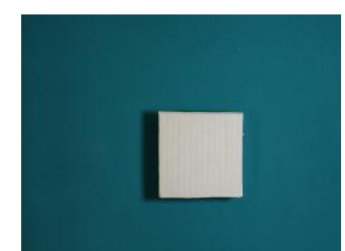

(b) Illumination 2

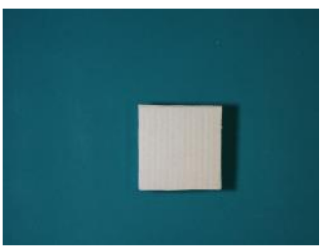

(d) Illumination 4
Figure 2: For images captured by the camera in Figure 1.

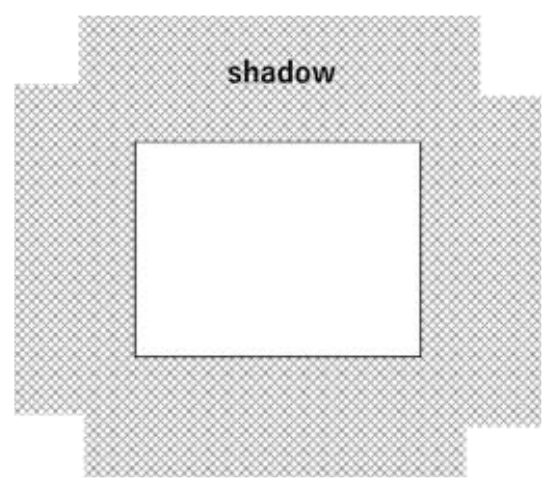

Figure 3:An image synthesized by four images in Figure 2.

In this study, we measure the size and position of cardboard boxes by the use of four illuminations and a camera. This method can correctly measure them even when cardboard boxes are overlapped.

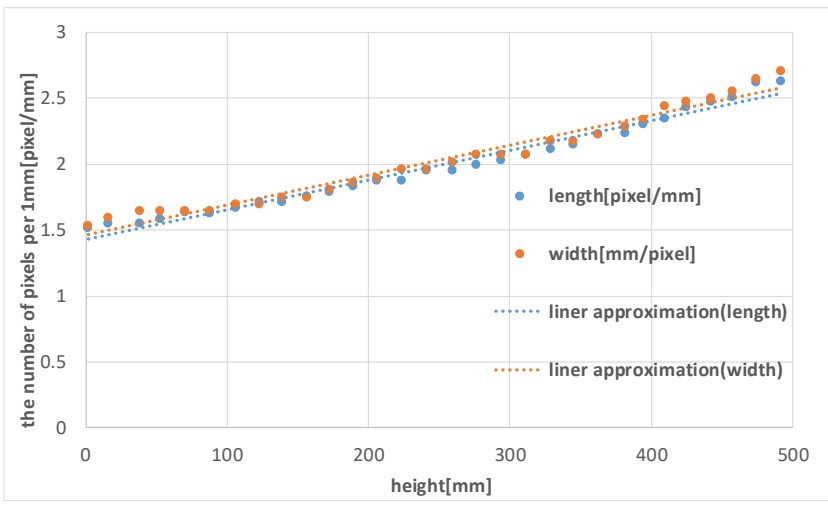

Figure 4: Influence of height on the number of pixels per $\mathbf{1}[\mathbf{m m}]$ in camera.

Figure 1 shows the outline of the system used in this study. The four illuminations are irradiated sequentially from illumination (1) to illumination (4). Figure 2 shows the image when each illumination is on. As understood from this figure, the shadow position differs from illumination (1) to illumination (4). By overlaying four images, we can show a shadow around the cardboard box. The schematic diagram is shown in Figure 3.

\subsection{Preprocessing in image processing}

As preprocessing of image processing, noise removal, image enhancement, binarization were performed.

A filter with a Gaussian distribution is used to remove image noise. In this study, the standard deviation representing the size of the filter is set to 3. A contrast emphasis is practiced in order to make a part of cardboard box part and a part of shadow clear. The histogram used in the contrast emphasis is extended to 0 to 255 in brightness. Binarization can extract the region of interest with an appropriate threshold value. In this study, the threshold is set to 100 .

\section{Experiment}

\subsection{Outline of experiment system}

Even if the size of the object is the same, as the object approaches the camera, the object on the screen is projected larger. In this experiment, since the camera is attached to the top of the system, the image size varies depending on the height of the object. In this experiment, we changed the height (z) of a box (200 mm (x) x $150 \mathrm{~mm}(\mathrm{y}) *(\mathrm{z})$ ) from 1.5 $\mathrm{mm}$ to $500 \mathrm{~mm}$, and measured the size per pixel at each height. The results are shown in Figure 4. The horizontal axis is the height $\mathrm{x}[\mathrm{mm}]$, and the vertical axis is the size $[\mathrm{mm} /$ pixel]. 


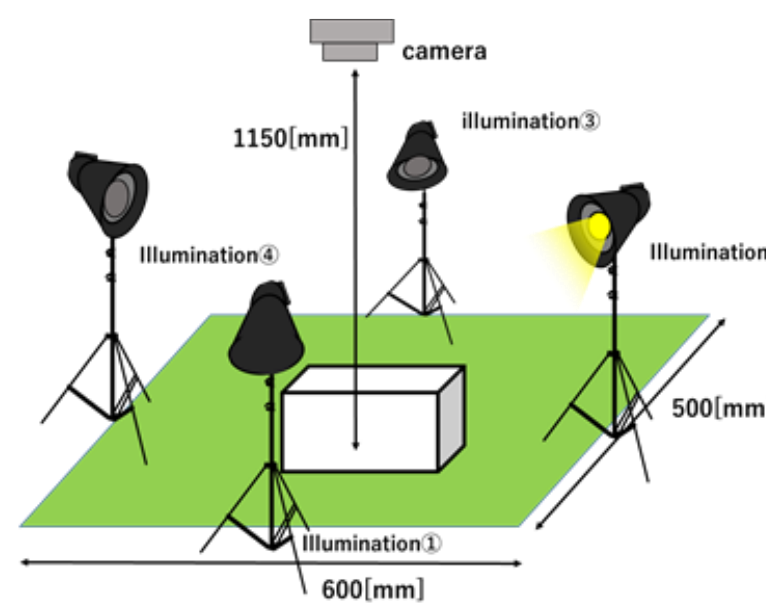

Figure 5: Position and the relationship of devices used in this experiment

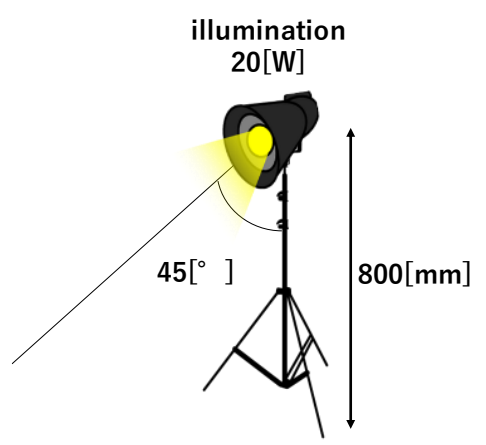

Figure 6: Condition of illumination used in this experiment.

\subsection{Measurement of cardboard box}

Figure 5 shows the positional relationship between the illuminations and the camera used in this experiment. Figure 6 shows the height and angle of the illumination.

\subsection{Experimental results}

The size of the four types of cardboard boxes used in the experiment is shown in Figure 7. As described in Section 2, in this experiment, the illuminations are irradiated from four directions. As the example is shown in Figure 2 mentioned above.

Figure 8 shows the image after the contrast adjustment of Figure 2, and Figure 9 shows the composite image of Figure 8. It is converted to grayscale as shown in Figure 10. As shown in Figure 11, it is smoothed by Gaussian filtering. It is binarized, and edge is extracted as shown in Figure 12. As a result, the size is detected as shown in Figure 13. The green line in the figure shows the size of the extracted box.

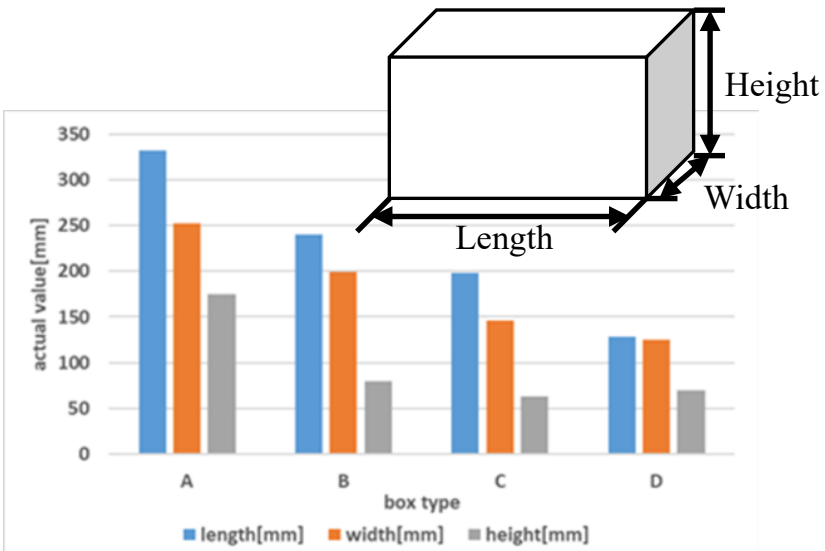

Figure 7: The sizes of the four types of cardboard boxes used in this experiment.

The sizes of the cardboard boxes A, B, C and D are measured by the same procedure.

The results are shown in Table 1. As seen from this table, the measured value is almost the same as the theoretical value. Although the error of box $\mathrm{A}$ is the largest, it is $4 \%$ at the maximum.

Next, when a cardboard box is placed on another cardboard box, the size and position of the upper box are measured. Here, we assume that the size of the upper box is larger than that of the lower box. The example is shown in Figure 14. It shows images when four illumination are irradiated to the box.

Figure 15 shows the detected box image. As seen from this figure, despite that the colors of the two boxes are the same, the upper box is correctly detected. Multiple cardboard boxes were measured in the same procedure. The results are shown in Table 2. Table 2 shows the measured values and theoretical values. As understood from this table, the largest error is $15 \%$ at Number 6 . Otherwise, it is about $5 \%$ at most.

From the above, it is possible to detect the size and position of the box almost exactly by this method. However, if the box above is larger than the box below it may not be detected. It is also necessary to optimize lighting conditions. In the future, in order to raise the detection rate more, image processing on video movie will be effective. These should be improved in the future. 


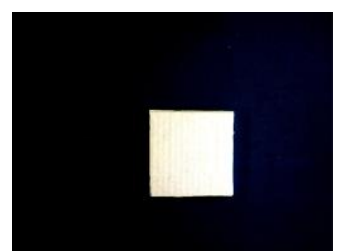

(a) illumination 1

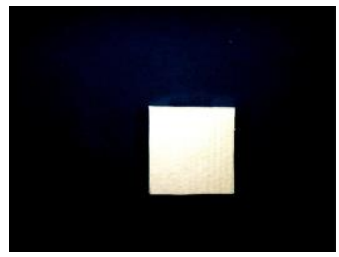

(c) illumination 3

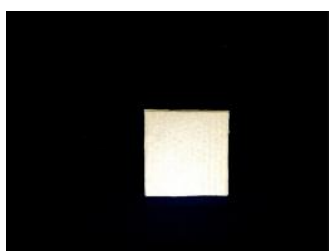

(b) illumination2

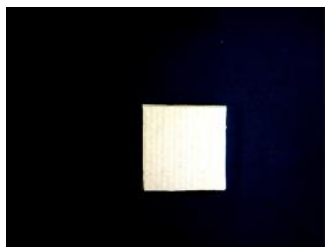

(d) illumination4
Figure 8: Enhancement processing of Figure 2.

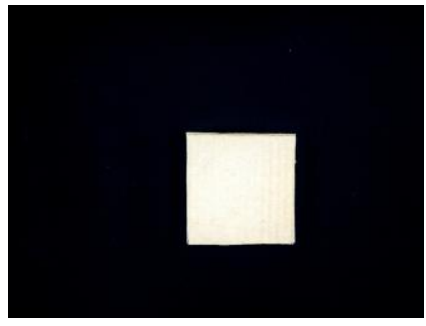

Figure 9: An image synthesized by four images i Figure 8.

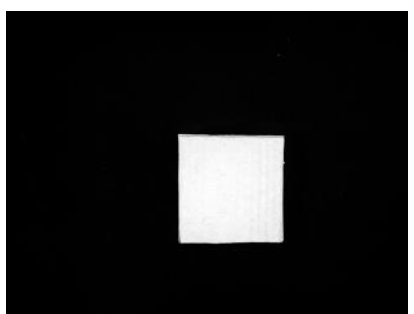

Figure 10: The image converted to gray scale in Figure 9.

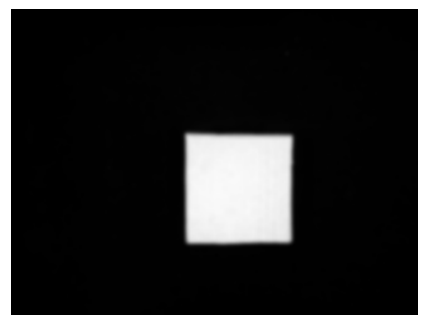

Figure 11: The image converted by Gaussian filtering in Figure 10

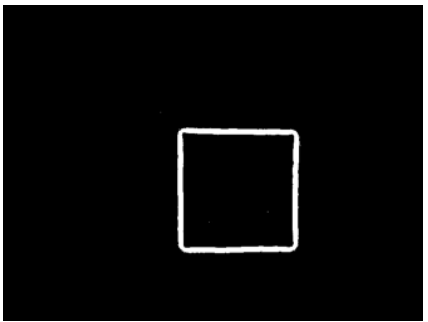

Figure 12: Binarized and edge extraction of Figure 11.

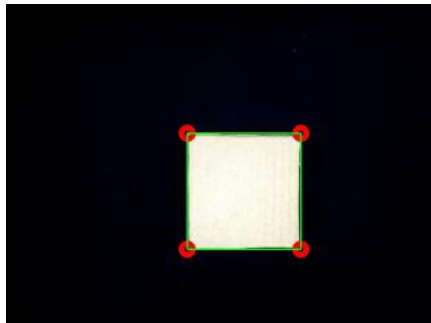

Figure 13: Detected size (green line).

Table 1 The measured size of cardboard boxes in this experiment.

\begin{tabular}{|c|c|c|c|c|c|c|}
\hline & & & \multicolumn{2}{|c|}{$\begin{array}{c}\text { measured } \\
\text { value }\end{array}$} & \multicolumn{2}{c|}{$\begin{array}{c}\text { theoretical } \\
\text { value }\end{array}$} \\
\hline number & $\begin{array}{c}\text { box } \\
\text { type }\end{array}$ & $\begin{array}{c}\text { height } \\
{[\mathrm{mm}]}\end{array}$ & $\begin{array}{c}\text { length } \\
{[\mathrm{mm}]}\end{array}$ & $\begin{array}{c}\text { width } \\
{[\mathrm{mm}]}\end{array}$ & $\begin{array}{c}\text { length } \\
{[\mathrm{mm}]}\end{array}$ & $\begin{array}{c}\text { width } \\
{[\mathrm{mm}]}\end{array}$ \\
\hline No.1 & A & 175 & 332 & 252 & 319 & 244 \\
\hline No.2 & B & 80 & 240 & 199 & 235 & 193 \\
\hline No.3 & C & 63 & 198 & 146 & 193 & 145 \\
\hline No.4 & D & 70 & 128 & 125 & 128 & 127 \\
\hline
\end{tabular}

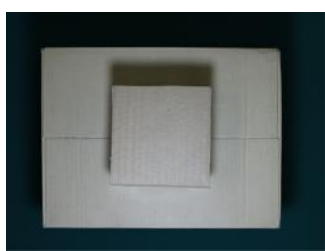

(a) Illumination 1

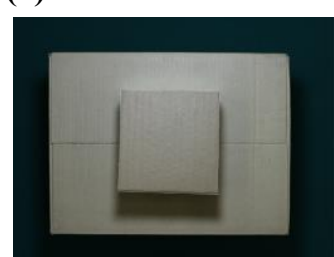

(c) Illumination 3

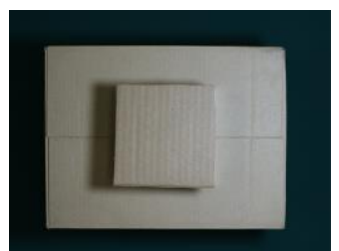

(b) Illumination 2

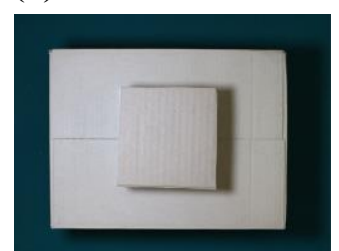

(d) Illumination 4
Figure 14: Images obtained by irradiating light from four directions on the box type $A$ and $D$. 


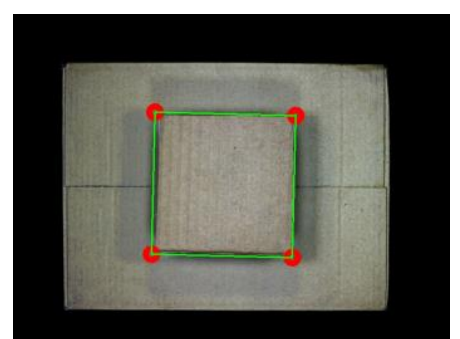

Figure 15: Detected size (green line).

Table 2 The sizes of the four cardboard boxes detected in case of overlapped boxes.

\begin{tabular}{|c|c|c|c|c|c|c|}
\hline & \multicolumn{2}{|c|}{ box type } & \multicolumn{2}{c|}{$\begin{array}{c}\text { upper side box } \\
\text { measured value }\end{array}$} & \multicolumn{2}{c|}{$\begin{array}{c}\text { upper side box } \\
\text { theoritical value }\end{array}$} \\
\hline Number & $\begin{array}{c}\text { Lower } \\
\text { side } \\
\text { box }\end{array}$ & $\begin{array}{c}\text { Upper } \\
\text { side } \\
\text { box }\end{array}$ & $\begin{array}{c}\text { Length } \\
{[\mathrm{mm}]}\end{array}$ & $\begin{array}{c}\text { Width } \\
{[\mathrm{mm}]}\end{array}$ & $\begin{array}{c}\text { Length } \\
{[\mathrm{mm}]}\end{array}$ & $\begin{array}{c}\text { Width } \\
{[\mathrm{mm}]}\end{array}$ \\
\hline No.1 & A & B & 240 & 199 & 229 & 187 \\
\hline No.2 & A & C & 198 & 146 & 194 & 142 \\
\hline No.3 & A & D & 128 & 125 & 128 & 127 \\
\hline No.4 & B & C & 198 & 146 & 196 & 143 \\
\hline No.5 & B & D & 128 & 125 & 128 & 126 \\
\hline No.6 & C & D & 128 & 125 & 151 & 134 \\
\hline
\end{tabular}

\section{Conclusions}

Recently, the introduction of automatic guided vehicles has increased in warehouses. However, it often drops luggage. To prevent this, the technique to measure the position and size of luggage is required in the vehicle. However, it is difficult to prepare it at low cost. As a result, the measuring equipment for luggage has not been introduced. In order to solve this problem, it is useful to use an image processing device. However, in case that the colors of the luggage are similar, the recognition rate becomes extremely low.

In order to solve this problem, we propose a method to detect the size and position of a cardboard box using four lights. In this study, four lights are attached on a vehicle. By overlapping images from 4 directions, there is a shadow even when cardboard boxes are overlapped. This easily extracts the edge of the cardboard box. From the extracted edge, we can detect the size of the cardboard box. For the validity of our method, we practiced experiments. The cardboard boxes are all the same color. As a result, even if the cardboard box is overlapped, we can detect the size and positon of a box correctly.

In future work, in order to raise the detection rate more, it is necessary to optimize lighting conditions and image processing on video movie should be utilized.

\section{References}

(1) Yoshizawa toru (2006), “Advanced 3D measurement using light” , Asakura-shuppan, pp.4-136

(2) P.J. Besl and Neil D. McKay (1992), "A method for registration of 3-D shapes", IEEE, Volume14,pp.239256

(3) Xiuhua Li and Wenfu Wu (2013), "Research on the Key Technique of Three-Dimensional Measurement Based on Laser Ranging”, IEEE, pp.3-6

(4) Xiaofeng Lian and Zaiwen Liu (2008), "Reconstructing indoor environmental 3D model using laser range scanners and omnidirectional camera", IEEE, pp.16401644

(5) Sheikh Faridul Hasan and Rafi Md. Najmus Sadat (2006), "A Precise and Low Complexity Distance and Size Measurement of Circular Objects from Camera Position using Still Images”, IEEE, pp.439-442

(6) Yasir M Mustafah, and Rahizall Noor (2012), "Stereo vision images processing for real-time object distance and size measurements", IEEE, pp.659-663 